\title{
GEARBOX FAULT CLASSIFICATION BY USING FREQUENCY BASED FEATURE EXTRACTION
}

\author{
Murat BAŞARAN 1, *iD , Mehmet FİDAN ${ }^{1}$ (i) \\ ${ }^{1}$ Vocational School of Transportation, Eskişehir Technical University, Eskisehir, Turkey
}

\begin{abstract}
Gearboxes are the fundamental elements of rotational systems to provide speed adjustment ratios from a rotating power source to another. In industrial applications, the existence of any kind of faults in rotational systems may be hazardous unless the early detection and maintenance procedures are applied. Incipient types of faults such as a few chipped or worn teeth at the gearbox mechanism may deteriorate and cause the maladjustment of the rotation and even the mechanism may stop to rotate which may cause loss of the production. Preventive maintenance strategies such as monitoring of the vibration signals and comparison of the frequency domain irregularities with normal operation case with healthy gearbox elements is essential to ensure safe and accurate rotational speed transmission in industrial systems. In this work, frequency domain characteristics of three different pinion conditions; healthy, a chipped tooth, and three consequent worn teeth are analyzed, and frequency domain features are proposed for classification of the pinion state. Proposed features obtained from the statistical properties of the coefficients of third level Wavelet packet decomposition. After feature extraction process, classification of the gear condition is made with different Support Vector Machine based classifiers and significant classification success observed with the proposed technique.
\end{abstract}

Keywords: Fault classification, Gearbox, Preventive maintenance, Support vector machine, Wavelet packet decomposition

\section{INTRODUCTION}

Gearboxes are the fundamental elements of rotational systems to provide speed adjustment ratios from a rotating power source to another. In industrial applications, the existence of any kind of faults in rotational systems may be hazardous unless the early detection and maintenance procedures applied. In many industrial applications, gearbox systems usually provide speed adjustments of electric motors such as induction motors. Induction motors are the most widely used, simple and durable constructed, and cost-effective priced electric motors but the processes run by motors with a gearbox system may be critical. Therefore, the need for preventive maintenance is obvious since the process which is run by induction motors may be critical and unwanted stops may lead to other problems like financial and labor losses [1]. For these reasons, condition monitoring of the gears in the gearbox is an important aspect to ensure safe operation of the industrial processes.

Condition monitoring of gearbox systems is very important research topic for researchers work in gearbox development. The preventive maintenance strategy, which is mainly about the early detection of cracks or worn teeth in gears, is crucial for recognizing the indicators before the fault deteriorates. Then it is possible for the researchers are to recognize where the crack began and to decide about the reason for the gearbox fault. Consequently, the designers can take necessary precautions and apply appropriate steps in gearbox design to improve gearbox performance. Condition monitoring systems deal with various types of inputs that show fault indicators, for instance vibration, acoustic emission, temperature level, oil contamination analysis [2]. Especially systems based on vibration analysis is the most common and effective fault detection systems and they can be applied very well in industry by placing simple vibration sensors on gearboxes.

In literature, numerous frequency-based techniques have been applied to diagnose and classify faults in gears. Fast Fourier transform (FFT) is a well-known technique using frequency contents of a signal can

*Corresponding Author: muratb@eskisehir.edu.tr

Received: $30.10 .2020 \quad$ Published: 27.11.2020 
be found out. In analysis of the frequency spectrum of the vibration, Wavelet packet decomposition (WPD) technique provides finer frequency resolution for localization of the fault related components. In this work, first FFT is applied to the vibration signals to observe the distribution of the frequency contents of the obtained vibration signals from the gearbox test setup. Then, it is observed that lowfrequency components of the signals have more local peaks that show the indicators of the worn teeth faults in the gear. For this reason, to reach finer frequency resolution, discrete Wavelet packet decomposition (WPD) of level 3 is applied to the vibration signals. After applying this transform, the statistical properties of the obtained coefficients related to the node corresponding to $0-1250 \mathrm{~Hz}$ frequency interval are used as features for classification.

\section{MATERIALS AND FEATURE EXTRACTION METHOD}

\subsection{Experimental Data Set for Gear Fault Diagnosis}

In this study, publicly available dataset consists of radial vibration signals acquired in a gearbox setup which can be seen in Figure 1 is used [3,4]. The system includes an electric motor, a brake system and a gearbox which runs the electric motor with an adjusted speed reference. The helical gear provides a speed ratio of 7.33 with a pinion having 15 teeth and a wheel having 110 teeth in the gearbox. The gearbox is tested with three different gear conditions: healthy, a chipped tooth, and three consequent worn teeth in helical gears where the latter two can be seen in Figure 2.

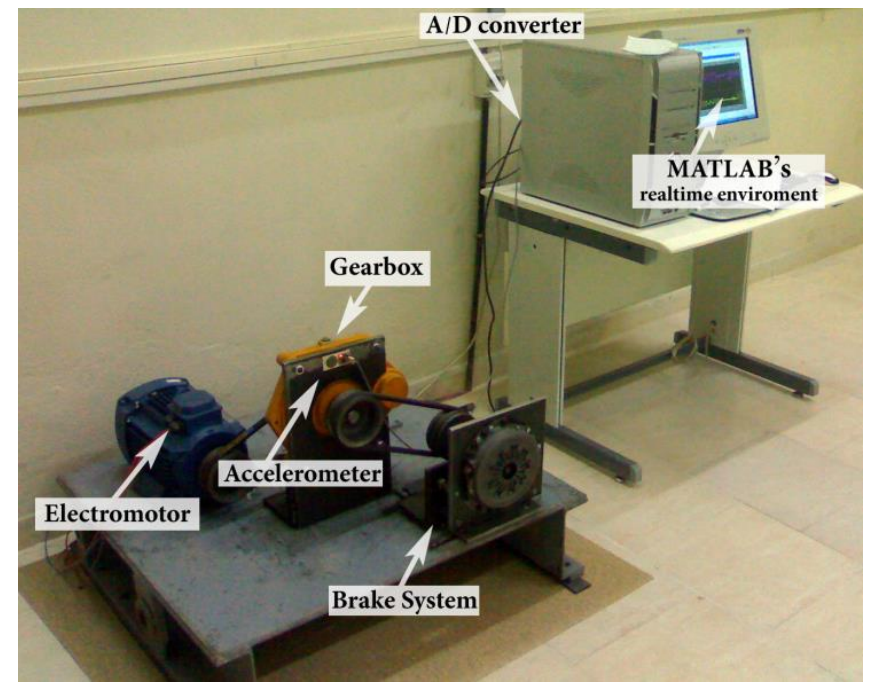

Figure 1. Zamanian's experimental gearbox test setup

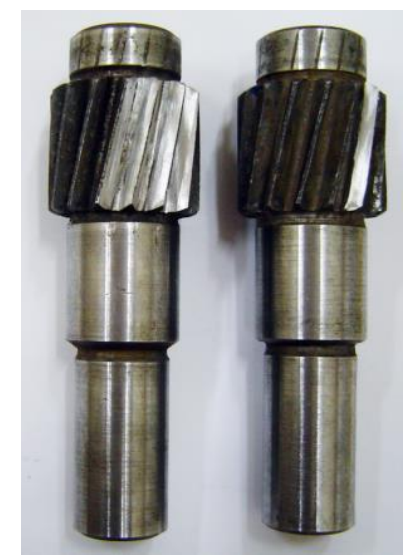

Figure 2. Gears with three consequent worn teeth and a chipped tooth 
The nominal speed of the rotation during experiments is $1420 \mathrm{rpm}$ and the vibration signals are recorded for a duration of 10 seconds via an accelerometer which has a sensitivity level of $100 \mathrm{mV} / \mathrm{g}$ placed onto the gearbox. Analog to digital conversion of the vibration signals is acquired by a data acquisition card with 12-bit vertical resolution at $10 \mathrm{kHz}$ sampling frequency which consequently leads to data files of length 100,000 samples for each gear condition.

\subsection{Fast Fourier Transform of the Vibration Signals}

In this work, first FFT is applied to the vibration signals to observe the distribution of the frequency contents of the obtained vibration signals from the gearbox test setup. When FFT is applied, it is seen that high frequency components of all fault types are insignificant but the low frequency components alter much. For instance, when we compare the healthy gear condition to others, it contains fewer local peaks. Furthermore, if the comparison done between a chipped tooth case with three worn teeth case, latter one includes more local peaks in its frequency spectrum. These frequency spectra related to these three conditions are given in Figures 3-5. Due to the FFT spectra, it seemed more beneficial to apply third level of discrete Wavelet packet decomposition to focus on the low-frequency part between 0-1250 $\mathrm{Hz}$ in order to reflect the fault characteristics better.

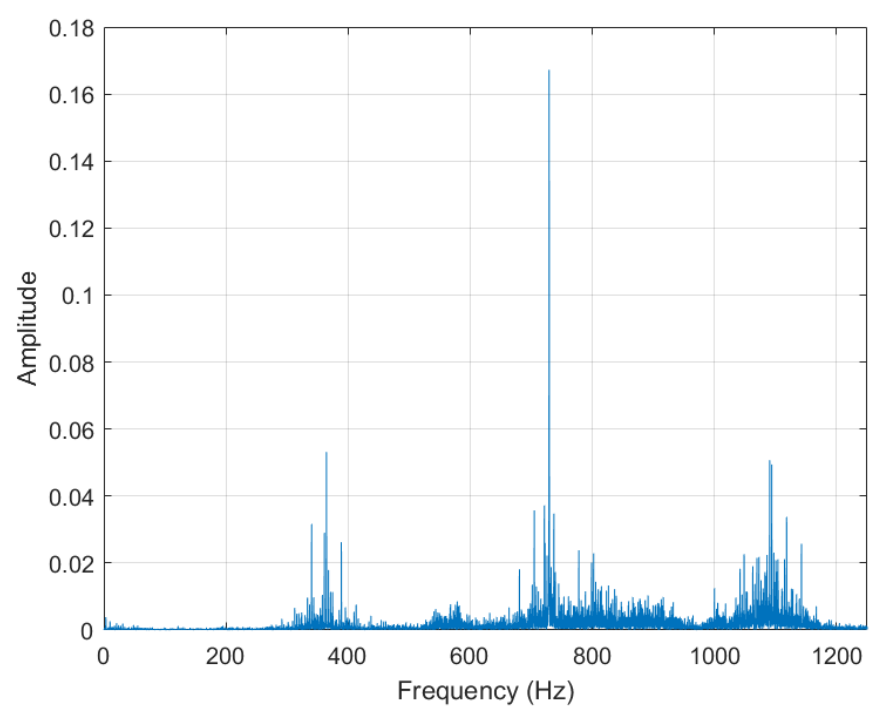

Figure 3. FFT spectrum of vibration signal of healthy gear condition

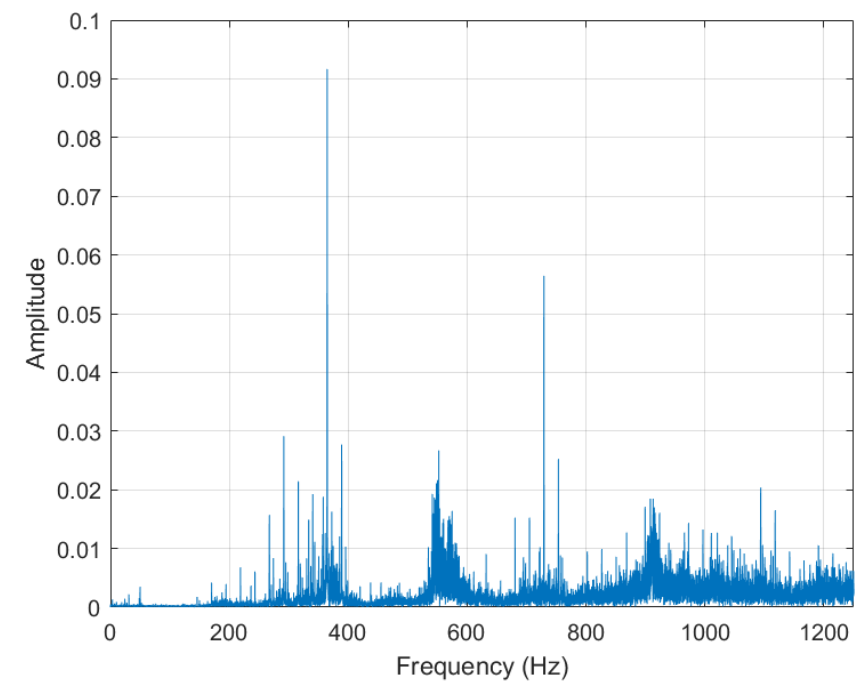

Figure 4. FFT spectrum of vibration signal of a chipped tooth gear condition 


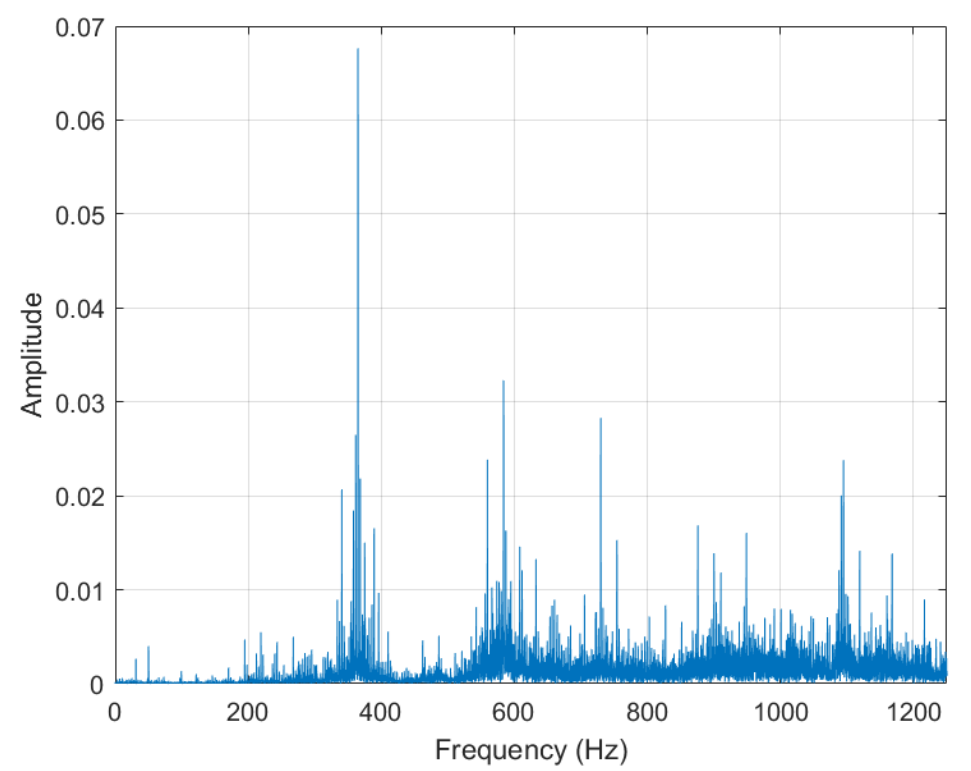

Figure 5. FFT spectrum of vibration signal of three worn teeth gear condition

\subsection{Wavelet Packet Decomposition of the Vibration Signals}

In analysis of the frequency spectrum of vibration signals, Wavelet packet decomposition (WPD) technique provides finer frequency resolution for localization of the indicators of faults. Time localization ability and multiresolution analysis properties make WPD superior for the fault detection. When applying wavelet transform to the original signal, it is divided into two parts, high frequency and low frequency part which are called the approximation and the detail, respectively. Unlike multiresolution analysis, where only low frequency parts are decomposed iteratively, in WPD, both approximation and detail spaces are decomposed further until the desired frequency resolution is reached [5]. In this study, two simple wavelet types, Haar and Daubechies-2 (db2) are used in WPD.

\subsection{Feature Extraction from Wavelet Packet Decomposition Coefficients}

The digitized vibration signals recorded for different gear conditions are used for feature extraction. First, data length of 100,000 samples are divided into 100 equal parts, resulting size of 1000 samples corresponding to 0.1 seconds of data record. By using this partitioning, 100 data parts extracted from each experiment recording. After applying $3^{\text {rd }}$ level of WPD to these data parts, four statistical properties of WPD coefficients are used to build up feature vectors. These four fundamental statistical parameters are mean, standard deviation, skewness, and kurtosis of each of the WPD coefficients of the node corresponding to the frequency interval of $0-1250 \mathrm{~Hz}$. Hence, from each recorded gear condition data, 100 feature vectors with 4 elements were obtained for each wavelet type used in WPD. Then, these two combined feature sets are used for classification by using Support Vector Machine (SVM) based classifiers.

\subsection{Support Vector Machine Based Classification}

Support Vector Machine (SVM) is a classification method, firstly proposed by Vapnik and Lerner in 1963, originally proposed for two class problems [6]. SVM aims to find the best hyperplanes that distinguish data samples of one class from the data samples of the other class. In the original SVM, this separating hyperplane is assumed as linear. The best separating hyperplane for SVM obtains the largest margin between the two classes. Support vectors are samples of the classes closest to the separating hyperplane and these samples are on the border of the respective classes. The distance between the 
planes passing through the support vectors and parallel to the separating hyperplane determines the length of the margin.

Although the original SVM was designed as a linear classifier, it is insufficient in most of the real-life classification problems. In practice, the hyperplane between two classes could be indented or curved, which can be defined only by a nonlinear function. To overcome this problem, a kernel trick is first proposed in 1964 [7]. Then the proposed kernel trick is improved and applied it to SVM in 1992 [8]. The resulting nonlinear classifier was replaced by a nonlinear kernel function of each data sample. Thus, a maximum-margin hyperplane fit into a transformed feature space is achieved. Although the classifier has a linear hyperplane in the transformed feature space, it shows nonlinear property in the original sample space. Besides original linear SVM, the mostly used nonlinear kernel types can be listed as quadratic, cubic and gaussian. In this work, classification of fault types is realized with the mentioned SVM classifier types and significant classification success are observed.

\section{COMPARATIVE RESULTS}

After obtaining feature sets from wavelet coefficients by selecting two different wavelet types in WPD, classification is made by using MATLAB's classification learner tool with four different SVM-based classifiers. Classification performances of SVM classifiers with features created with two different wavelet selection are given in Table 1.

Table 1. Classification performances of SVM classifier with features created with 2 different wavelet selection

\begin{tabular}{|c|c|c|c|c|}
\hline Classifier & $\begin{array}{c}\text { Linear } \\
\text { SVM }\end{array}$ & $\begin{array}{c}\text { Quadratic } \\
\text { SVM }\end{array}$ & $\begin{array}{c}\text { Cubic } \\
\text { SVM }\end{array}$ & $\begin{array}{c}\text { Gaussian } \\
\text { SVM }\end{array}$ \\
\hline Haar Wavelet & $89.7 \%$ & $90.3 \%$ & $90.3 \%$ & $92.7 \%$ \\
\hline Db-2 Wavelet & $90.7 \%$ & $91.0 \%$ & $90.0 \%$ & $92.3 \%$ \\
\hline
\end{tabular}

In order to observe misclassified instances, it is necessary to construct confusion matrices for each classifier. Therefore, Tables 2-9 are given to observe classification performances in detail.

Table 2. Confusion matrix for Linear SVM classifier (Haar Wavelet)

\begin{tabular}{|c|c|c|c|c|}
\hline & \multicolumn{3}{|c|}{ predicted class } \\
\cline { 2 - 5 } & & a chipped tooth & healthy gear & three worn teeth \\
\cline { 2 - 5 } & a chipped tooth & 90 & 6 & 4 \\
\cline { 2 - 5 } & healthy gear & 2 & 90 & 8 \\
\cline { 2 - 5 } & three worn teeth & 0 & 11 & 89 \\
\hline
\end{tabular}

Table 3. Confusion matrix for Quadratic SVM classifier (Haar Wavelet)

\begin{tabular}{|c|c|c|c|c|}
\hline \multirow{2}{*}{} & & \multicolumn{3}{|c|}{ predicted class } \\
\cline { 2 - 5 } & & a chipped tooth & healthy gear & three worn teeth \\
\cline { 2 - 5 } & a chipped tooth & 89 & 9 & 2 \\
\cline { 2 - 5 } & healthy gear & 4 & 90 & 6 \\
\cline { 2 - 5 } & three worn teeth & 1 & 7 & 92 \\
\hline
\end{tabular}


Table 4. Confusion matrix for Cubic SVM classifier (Haar Wavelet)

\begin{tabular}{|c|c|c|c|c|}
\hline \multirow{5}{*}{ 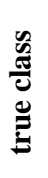 } & & \multicolumn{3}{|c|}{ predicted class } \\
\hline & & a chipped tooth & healthy gear & three worn teeth \\
\hline & a chipped tooth & 89 & 8 & 3 \\
\hline & healthy gear & 4 & 88 & 8 \\
\hline & three worn teeth & 1 & 5 & 94 \\
\hline
\end{tabular}

Table 5. Confusion matrix for Gaussian SVM classifier (Haar Wavelet)

\begin{tabular}{|c|c|c|c|c|}
\hline \multirow{2}{*}{} & & \multicolumn{3}{|c|}{ predicted class } \\
\cline { 2 - 5 } & & a chipped tooth & healthy gear & three worn teeth \\
\cline { 2 - 5 } & a chipped tooth & 92 & 3 & 5 \\
\cline { 2 - 5 } & healthy gear & 4 & 92 & 4 \\
\cline { 2 - 5 } & three worn teeth & 0 & 6 & 94 \\
\hline
\end{tabular}

Table 6. Confusion matrix for Linear SVM classifier (db2 Wavelet)

\begin{tabular}{|c|c|c|c|c|}
\hline \multirow{2}{*}{} & & \multicolumn{3}{|c|}{ predicted class } \\
\cline { 2 - 5 } & & a chipped tooth & healthy gear & three worn teeth \\
\cline { 2 - 5 } & a chipped tooth & 91 & 7 & 2 \\
\cline { 2 - 5 } & healthy gear & 1 & 90 & 9 \\
\cline { 2 - 5 } & three worn teeth & 0 & 9 & 91 \\
\hline
\end{tabular}

Table 7. Confusion matrix for Quadratic SVM classifier (db2 Wavelet)

\begin{tabular}{|c|c|c|c|c|}
\hline & & \multicolumn{3}{|c|}{ predicted class } \\
\cline { 2 - 5 } & & a chipped tooth & healthy gear & three worn teeth \\
\cline { 2 - 5 } & a chipped tooth & 92 & 4 & 4 \\
\cline { 2 - 5 } & healthy gear & 2 & 89 & 9 \\
\cline { 2 - 5 } & three worn teeth & 1 & 7 & 92 \\
\hline
\end{tabular}

Table 8. Confusion matrix for Cubic SVM classifier ( $\mathrm{db} 2$ Wavelet)

\begin{tabular}{|c|c|c|c|c|}
\hline \multirow{2}{*}{} & & \multicolumn{3}{|c|}{ predicted class } \\
\cline { 2 - 5 } & & a chipped tooth & healthy gear & three worn teeth \\
\cline { 2 - 5 } & a chipped tooth & 90 & 6 & 4 \\
\cline { 2 - 5 } & healthy gear & 3 & 87 & 10 \\
\cline { 2 - 5 } & three worn teeth & 1 & 6 & 93 \\
\hline
\end{tabular}

Table 9. Confusion matrix for Gaussian SVM classifier (db2 Wavelet)

\begin{tabular}{|c|c|c|c|c|}
\hline \multirow{2}{*}{} & & \multicolumn{3}{|c|}{ predicted class } \\
\cline { 2 - 5 } & & a chipped tooth & healthy gear & three worn teeth \\
\cline { 2 - 5 } & a chipped tooth & 92 & 5 & 3 \\
\cline { 2 - 5 } & healthy gear & 1 & 93 & 6 \\
\cline { 2 - 5 } & three worn teeth & 2 & 6 & 92 \\
\hline
\end{tabular}




\section{CONCLUSIONS}

In this work, frequency domain characteristics of three different pinion conditions of the gearbox vibration data; healthy, a chipped tooth, and three consequent worn teeth are analyzed, and frequencybased features are proposed for classification of the gearbox condition. Proposed features obtained from the statistical properties of the coefficients of third level Wavelet packet decomposition. After feature extraction process, classification of the gear condition is made with different Support Vector machinebased classifiers and significant classification success above $90 \%$ observed with the proposed technique.

\section{REFERENCES}

[1] Kumar RS, Ray K and Kumar KV. Fault Diagnosis of Industrial Drives using MCSA Techniques, Paper Presented at the 2009 International Conference on Control, Automation, Communication and Energy Conservation, 2009.

[2] Večeř P, Marcel Kreid, and Šmíd R. Condition Indicators for Gearbox Condition Monitoring Systems, Acta Polytechnica vol. 45, no. 6, 2005.

[3] Zamanian AH and Ohadi A, Gear Fault Diagnosis Based on Gaussian Correlation of Vibrations Signals and Wavelet Coefficients, Applied Soft Computing, vol. 11, no. 8, pp. 4807-4819, 2011.

[4] Zamanian AH and Ohadi A.Gearbox Fault Detection Through PSO Exact Wavelet Analysis and SVM Classifier, in 18th Annual International Conference on Mechanical EngineeringISME2010, 2010.

[5] Akansu AN, Haddad PA, Haddad RA and Haddad PR. Multiresolution Signal Decomposition: Transforms, Subbands, and Wavelets, Academic press, 2001.

[6] Vapnik V and Lerner AJ. Generalized Portrait Method for Pattern Recognition, Automation and Remote Control, vol. 24, no. 6, pp. 774-780, 1963.

[7] Aiserman M, Braverman, EM and Rozonoer L, Theoretical Foundations of the Potential Function Method in Pattern Recognition, Avtomat i Telemeh, vol. 25, no. 6, pp. 917-936, 1964.

[8] Boser BE, Guyon, IM and Vapnik V. A Training Algorithm for Optimal Margin Classifiers, In Proceedings of the Fifth Annual Workshop on Computational Learning Theory, pp. 14. 